\title{
Implementing evidence-based guidelines for managing depression in elderly patients: a Norwegian perspective
}

\author{
E. Aakhus ${ }^{1,2 *}$, S. A. Flottorp ${ }^{2,3}$ and A. D. Oxman ${ }^{2}$ \\ ${ }^{1}$ Research Centre for Old Age Psychiatry, Innlandet Hospital Trust, 2312 Ottestad, Norway \\ 2 The Norwegian Knowledge Centre for the health Services, N-0130, Oslo, Norway \\ ${ }^{3}$ Department of Public Health and Primary Health Care, University of Bergen, Norway
}

\begin{abstract}
Depression in the elderly is common and exhibits a distinctive phenomenology, due to neurobiological, physiological, psychological and social changes related to ageing. Most elderly with depression are managed in primary health care. Although the number of scientific publications related to geriatric psychiatry has increased, there are still important gaps. Implementation of evidence-based guidelines for managing depression in primary care has had limited success, but has led to improvements compared to standard care. It is logical that the determinants (barriers and enablers) of implementing depression guidelines can be identified and can guide the selection of more effective implementation strategies that are tailored to address those determinants. We are testing that logic as part of a multinational implementation research project called 'Tailored Implementation for Chronic Diseases' (TICD). Our focus in Norway is on the management of depression in the elderly in primary care. We will identify the determinants of implementing evidence-based recommendations using various methods and comparing those methods. We will then use different methods to match the implementation interventions to the identified determinants and compare those methods. Finally, we will evaluate the resulting tailored implementation strategy in a randomized trial.
\end{abstract}

First published online 22 May 2012

Key words: Barriers, depression, elderly, enablers, implementation, primary care.

\section{Introduction}

Psychiatric disorders in old age exhibit a distinctive phenomenology. Depression, delirium and anxiety disorders are the most common clinical presentations in addition to the different dementia disorders. The evaluation of psychiatric symptoms as prodromal or early stage presentation of dementia is one of the most challenging tasks for the geriatric psychiatrist. The complexity of psychiatric disorders in old age is often a result of medical co-morbidity and polypharmacy. Although geriatric psychiatry is a discipline within the specialist health care system, most elderly with psychiatric disorders in Norway are managed in primary health care. Furthermore, elderly with depression wish to be treated by their primary care physician according to international data (Oxman et al. 2003). Unpublished data from a survey conducted by the Norwegian Psychological Association confirmed this finding, indicating that over $60 \%$ of the adult population prefer to be treated by their general practitioner or by primary care services (Norsk

\footnotetext{
* Address for correspondence: Eivind Aakhus, Research Centre for Old Age Psychiatry, Innlandet Hospital Trust, 2312 Ottestad, Norway (Email: eivind.aakhus@gmail.com)
}

Psykologforening \& tnsGallup, 2004), and only approximately $20 \%$ of elderly would prefer to be treated by a psychologist or a psychiatrist when faced with psychological problems. Geriatric psychiatry is a clinical discipline for which the body of evidence-based treatment options is growing (Bartels, 2005). There is moderate to high-quality evidence for several recommendations (Buchanan et al. 2006; NICE, 2010) and health care providers need access to clear recommendations that are informed by the best available evidence (Graham et al. 2011). As part of a multinational project called 'Tailored Implementation for Chronic Diseases' (TICD) (Wensing, 2011), we will identify the determinants (barriers and enablers) of the implementation of evidence-based recommendations for the management of depression in the elderly and we will tailor an implementation strategy to address those determinants in Norwegian primary health care.

\section{Depression in the elderly}

Depression is one of the most common psychiatric disorders in the elderly. Approximately $15 \%$ of the elderly suffer from some degree of depression, and the prevalence increases with increasing age (Skoog, 2004). 
Recent data from the Norwegian epidemiological cohort study HUNT3 identified a markedly higher prevalence of depression in age groups above 75 years (Solhaug et al. 2012) as compared with younger people (45-74 years), and a highly significant increase in the proportion of depression among people older than 75 years, when compared with their own depression rates at a younger age (as compared with data from HUNT2) (Stordal et al. 2003). Within the group of elderly, the prevalence of depression varies, depending on the patient's medical and psychiatric co-morbidity and their level of care (Rosenvinge \& Rosenvinge, 2003).

Relapse rates are higher among the elderly, as compared with younger adults, even if the treatment response is similar (Mitchell \& Subramaniam, 2005).

The elderly suffering from depression have a higher mortality risk than healthy elderly (Adamson et al. 2005). A score of six or higher on the 15-item Geriatric Depression Scale is associated with a $27 \%$ relative increase in the risk of dying within 3 years compared with those with a score below six (adjusting for chronic illness, disability, cognition, drinking and smoking). Depression in the elderly is often chronic. Cole et al. (1999) reported in a systematic review that $33 \%$ of depressed elderly still exhibited depressive symptoms at a 24-month follow-up, whereas $21 \%$ had deceased. Only 33\% were well. Denihan et al. (2000) reported in a 3-year follow-up study of 127 community dwelling elderly with depression that $35 \%$ had persistent or recurrent depression and $25 \%$ had other mental illness. Only 10\% had recovered completely, indicating that depression in the elderly, in a long-term perspective, carries a high risk of complications and chronicity. Licht-Strunk et al. (2007) reported that age was an independent risk factor for a negative prognosis. Cole \& Dendukuri (2003) reported in a meta-analysis that loss, sleep disturbance, functional decline, a previous episode of depression and female gender implied a higher risk for developing depression among elderly. In a British study (Nelson et al. 2002), elderly patients with depression were likely to consult with their primary care physician, but only a small number of depressed patients (13\%) were treated with antidepressants. Mackenzie et al. (1999) found that general practitioners were less prepared to diagnose and treat elderly patients with depression compared with younger patients.

\section{Guidelines for depression in the elderly}

We have recently conducted a systematic review of guidelines for the management of depression, particularly focusing on the elderly with depression (not published yet). We compared 13 English or Scandinavian-language guidelines published in 2005 or later. We identified recommendations for the elderly with depression and compared these, using the Norwegian guideline for the management of depression in primary and specialist health care (Helsedirektoratet, 2009) as a comparator. We found that although the guidelines to a large extent were consistent, the number of recommendations regarding elderly patients varied. We assessed the quality of each guideline using AGREE II (AGREE Consortium, 2009). Overall the guidelines scored reasonably well. However, generally the guidelines scored low on 'Applicability', reflecting that the guidelines only to a limited degree described the determinants and implementation strategies to put the guideline into practice.

\section{Barriers to implementing guidelines for managing depression in the elderly}

A large number of factors can affect practice (e.g. organizational, professional and patient factors) (Cabana et al. 1999; Michie et al. 2005). The importance of these determinants of practice varies from recommendation to recommendation and can vary from setting to setting. For example, organizational capacity to implement changes are critical for implementing a recommendation for collaborative care, but may be unimportant for implementing a recommendation not to treat mild depression with antidepressants. On the other hand, the knowledge and skills of general practitioners are critical for implementing a recommendation to offer 'talking therapy' to patients with mild depression who do not improve. Patients' beliefs and attitudes to the recommended action may also be important. Consequently, it is necessary to consider and investigate the importance of specific factors for specific recommendations in specific contexts. The methods of investigating the importance of specific factors include interviews, focus groups and surveys with clinicians or patients.

One objective of the TICD project is to compare the use of different methods for doing this, since currently there is little evidence to inform decisions about how best to identify or assess the importance of barriers and enablers. A second objective of the TICD is to compare different methods for matching implementation strategies to identified determinants of practice. In the end, we will test the effects of implementation strategies that are tailored in this way in randomized trials.

Although several effective treatment options for the depressed elderly have been established (Steinman et al. 2007), barriers to the implementation of adequate 
treatment of elderly patients with depression are prevalent. In a review, Ell (2006) reported numerous barriers to depression care for the elderly, including:

(1) Barriers to detection of depression and inadequacy of treatment: elderly patients are less likely to receive an antidepressant treatment and less likely to receive an adequate course of psychotherapy.

(2) Patient barriers: elderly are less likely to report depressive symptoms or may be more likely to ascribe depressive symptoms to physical illness.

(3) Provider barriers: physicians may ascribe depression to the natural course of ageing and related physical illness and their attitudes and beliefs may affect depression management more than knowledge. Physicians also perceive treatment guidelines as insufficiently flexible for the variety of patients seen in primary care.

(4) Health system barriers: the absence of collaboration between health care providers in primary care and mental health services, and the shortage of trained professionals may limit the implementation of adequate care.

The implementation of guidelines for managing depression in primary care has led to improvements compared with standard practice, but whenever there have been improvements, they have been limited (Rollman et al. 2006). A plausible explanation for this is that the strategies that were used did not adequately address the barriers to implementing the guidelines or capitalize on the enablers. Logically, successful implementation of guidelines in primary care, or any other health care setting, requires identifying important barriers and enablers and using strategies that address these.

\section{The potential of tailored implementation strategies}

There is moderate quality evidence that tailoring interventions to take account of the barriers and enablers is effective compared with no intervention or dissemination of guidelines (Baker et al. 2010). However, there is a lack of evidence comparing tailored implementation strategies to non-tailored implementation strategies. There is also uncertainty about how best to identify the barriers and tailor interventions to address them. The TICD research project will help address that uncertainty by comparing different methods for identifying barriers and matching implementation interventions with those for five different chronic diseases in five European countries (depression in Norway, cardiovascular disease in the Netherlands, obesity in England, chronic obstructive pulmonary disease in Poland and multi-morbidity in Germany).
There are numerous methods for identifying barriers and enablers (Rainbird et al. 2006). We have used a Delphi process to assess the feasibility, comprehensiveness, validity, consistency, cost and relevance of different methods. Based on this process, we chose the following methods that will be used in all five countries to identify important barriers and enablers: brainstorming, focus groups, interviews and questionnaires with health care providers and interviews with patients or their relatives. We will compare these methods by randomizing study participants (health care professionals) with the different methods, in order to ensure that they are comparable, and by using different methods sequentially, to assess the added value of using methods that require more resources. In Norway, we will stratify health care professionals in five groups (physicians and nurses from primary and specialist health care and researchers) to ensure balanced representation of professionals and health care levels in each comparison group. We will present prioritized recommendations for managing depression in the elderly to the participants and discuss potential barriers and enablers with them. We will use a structured checklist to guide the focus groups and interviews. We will also conduct a survey based on the same checklist, including 250 health care professionals. We will analyse the data qualitatively and quantitatively, and we will compare the barriers and enablers identified using each method, the resources required to use each method and any problems, difficulties or positive experiences in applying the methods. The next step will be to match the implementation interventions to the identified barriers and enablers. This will also be performed in each participating country using several different methods and comparing those. Finally, we will conduct cluster-randomized trials of the tailored implementation strategies together with process evaluations in each of the five countries.

\section{Conclusion}

The TICD project will help address the paucity of evidence on the advantages and disadvantages of different methods of identifying important determinants of practice and selecting implementation strategies that take account of those. It will also provide a rigorous evaluation of an implementation strategy that is tailored to take account of specific barriers and enablers for implementing evidence-based recommendations for the management of depression in the elderly. Hopefully, the results of this research will provide much needed evidence to inform decisions about how to improve the quality of care provided to elderly patients with depression. 


\section{Acknowledgments}

The research leading to these results has received funding from the European Union Seventh Framework Programme (FP7/2007-2013) under Grant agreement no. 258837

\section{References}

Adamson JA, Price GM, Breeze E, Bulpitt CJ, Fletcher AE (2005). Are older people dying of depression? Findings from the Medical Research Council trial of the assessment and management of older people in the community. Journal of the American Geriatrics Society 53, 1128-1132.

AGREE Consortium (2009). AGREE Next Steps Consortium the AGREE II Instrument. Retrieved 3 March 2011 from http://www.agreetrust.org

Baker R, Camosso-Stefinovic J, Gillies C, Shaw EJ, Cheater F, Flottorp S, Robertson N (2010). Tailored interventions to overcome identified barriers to change: effects on professional practice and health care outcomes. Cochrane Database of Systematic Reviews CD005470.

Bartels SJ, Drake RE (2005). Evidence-based geriatric psychiatry: An Overview. Psychiatric Clinics of North America 28, 763-784.

Buchanan D, Tourigny-Rivard M-F, Cappeliez P, Frank C, Janikowski P, Spanjevic L, Malach FM, Mokry J, Flint A, Herrmann N (2006). National guidelines for seniors' mental health: the assessment and treatment of depression.

Canadian Journal of Geriatrics 9(Suppl. 2), S52-S58.

Cabana MD, Rand CS, Powe NR, Wu AW, Wilson MH, Abboud PA, Rubin HR (1999). Why don't physicians follow clinical practice guidelines? A framework for improvement. Journal of the American Medical Association 282, 1458-1465.

Cole MG \& Dendukuri N (2003). Risk factors for depression among elderly community subjects: a systematic review and meta-analysis. American Journal of Psychiatry 160, 1147-1156.

Cole MG, Bellavance F, Mansour A (1999). Prognosis of depression in elderly community and primary care populations: a systematic review and meta-analysis. American Journal of Psychiatry 156, 1182-1189.

Denihan A, Kirby M, Bruce I, Cunningham C, Coakley D, Lawlor BA (2000). Three-year prognosis of depression in the community-dwelling elderly. British Journal of Psychiatry 176, 453-457.

Ell K (2006). Depression care for the elderly: reducing barriers to evidence-based practice. Home Health Care Services Quarterly 25, 115-148.

Graham R, Mancher M, Wolman DM, Greenfield S, Steinberg E (2011). Clinical Practice We Can Trust. National Academies Press: Washington, DC.

Helsedirektoratet (2009). National Guidelines for the Management of Adults with Depression in Primary and Specialist Health Care. Helsedirektoratet: Oslo.

Licht-Strunk E, van der Windt DA, van Marwijk HW, de Haas M, Beekman AT (2007). The prognosis of depression in older patients in general practice and the community. A systematic review. Family Practice 24, 168-180.
Mackenzie CS, Gekoski WL, Knox VJ (1999). Do family physicians treat older patients with mental disorders differently from younger patients? Canadian Family Physician 45, 1219-1224.

Michie S, Johnston M, Abraham C, Lawton R, Parker D, Walker A (2005). Making psychological theory useful for implementing evidence based practice: a consensus approach. Quality and Safety in Health Care 14, 26-33.

Mitchell AJ, Subramaniam H (2005). Prognosis of depression in old age compared to middle age: a systematic review of comparative studies. American Journal of Psychiatry 162, 1588-1601.

Nelson T, Livingston G, Knapp M, Manela M, Kitchen G, Katona C (2002). Slicing the health service cake: the Islington study. Age and Ageing 31, 445-450.

NICE (2010). Depression. The NICE Guideline on the Treatment and Management of Depression in Adults. Updated edition. Royal College of Psychiatrist and British Psychological Society: London and Leicester.

Norsk Psykologforening \& TNS Gallup (2004) Survey on psychological health care services and treatment. Norwegian Psychological Association. Retrieved 27 January 2012 from (http://www.psykologforeningen.no).

Oxman TE, Dietrich AJ, Schulberg HC (2003). The depression care manager and mental health specialist as collaborators within primary care. American Journal of Geriatric Psychiatry 11, 507-516.

Rainbird K, Sanson-Fischer R, Buchan H (2006). Identifying Barriers to Evidence Uptakes. National Institute of Clinical Studies: Melbourne, Australia.

Rollman BL, Weinreb L, Korsen N, Schulberg HC (2006). Implementation of guideline-based care for depression in primary care. Administation Policy in Mental Health 33, 43-53.

Rosenvinge BH \& Rosenvinge JH (2003). Occurrence of depression in the elderly-a systematic review of 55 prevalence studies from 1990-2001. Tidsskrift for Den Norske Laegeforening 123, 928-929.

Skoog I (2004). Psychiatric epidemiology of old age: the H70 study - the NAPE lecture 2003. Acta Psychiatrica Scandinavica 109, 4-18.

Solhaug HI, Romuld EB, Romild U, Stordal E (2012). Increased prevalence of depression in cohorts of the elderly: an 11-year follow-up in the general population - the HUNT study. International Psychogeriatrics 24, 151-158.

Steinman LE, Frederick JT, Prohaska T, Satariano WA, Dornberg-Lee S, Fisher R, Graub PB, Leith K, Presby K, Sharkey J, Snyder S, Turner D, Wilson N, Yagoda L, Unutzer J, Snowden M, Late Life Depression Special Interest Project (SIP) Panelists (2007). Recommendations for treating depression in community-based older adults. American Journal of Preventive Medicine 33, 175-181.

Stordal E, Mykletun A, Dahl AA (2003). The association between age and depression in the general population: a multivariate examination. Acta Psychiatrica Scandinavica 107, 132-141.

Wensing M, Oxman A, Baker R, Godycki-Cwirko M, Flottorp SA, Szecsenyi J, Grimshaw J, Eccles M (2011). Tailored implementation for chronic diseases (TICD): A project protocol. Implementation Science 6, 103. 